\title{
European Hernia Society (EHS) guidance for the management of adult patients with a hernia during the COVID-19 pandemic
}

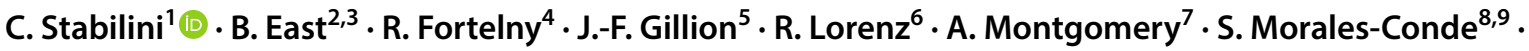 \\ F. Muysoms ${ }^{10} \cdot$ M. Pawlak ${ }^{11} \cdot$ W. Reinpold ${ }^{12} \cdot$ M. Simons ${ }^{13} \cdot$ A. C. de Beaux ${ }^{14}$
}

Received: 23 April 2020 / Accepted: 4 May 2020 / Published online: 15 May 2020

○ Springer-Verlag France SAS, part of Springer Nature 2020, corrected publication 2020

\section{General concepts}

The current COVID-19 pandemic has raised a number of problems never encountered before by the European Community. We are facing a difficult period in which a poorly known disease is being tackled with scarce resources by healthcare systems conceived largely for the elective treatment of benign and malignant conditions. These healthcare systems are having to retrain and conform to circumstances more similar to war scenarios. In this difficult and evolving situation, the European Hernia Society wants to share with its members some general concepts on abdominal wall surgery and highlight situations that merit attention.

As a general principle, it is paramount that every surgeon conforms to national and/or local guidelines concerning surgery and protection in the management of COVID-19 patients, suspected COVID-19 infections, and, indeed, all patients at this time.

Elective abdominal wall surgery has as its first aimimprovement of the patient's quality of life. Therefore, it is our opinion that it should be postponed during this time of

C. Stabilini

cesarestabil@hotmail.com

1 Department of Surgery, University of Genoa, Policlinico San Martino, Largo R. Benzi 8, 16132 Genoa, Italy

2 3rd Department of Surgery at 1st Faculty of Medicine at Charles University, Motol University Hospital, Prague, Czech Republic

3 2nd Faculty of Medicine, Charles University, Prague, Czech Republic

4 Department of General, Visceral and Oncologic Surgery, Wilhelminenspital, Vienna, Austria

5 Hôpital Privé d'Antony, 1 rue Velpeau, 92160 Antony, France

6 Hernia Center, CHIRURGEN 3+, Berlin, Germany

7 Department of Surgery, Lund University, Skåne University Hospital, Malmö, Sweden crisis. For inpatient surgery, the need for anesthesiologist and other healthcare input in addition to the technical equipment side cannot be sustained at present in several EU countries. For outpatient and day case surgery, even if performed under local anesthesia, the number of patients displaced and the risk of in-hospital infection make it difficult to justify. Thus, the focus of any hernia surgery at this time is largely the management of the emergency hernia, and also managing the current hernia waiting list and patients with a new hernia diagnosis. This is discussed in more detail.

The present document represents a clinical guidance from experts following published guidelines where available and adapting them to the current pandemic situation. Where evidence is lacking, we have made guidance based on expert opinion. We have adopted a practical, non-standardized style of question and answer, providing the evidence for our statements to inform on their reliability.

8 Unit of Innovation in Minimally Invasive Surgery, University Hospital Virgen del Rocio, University of Sevilla, Seville, Spain

9 Unit of General and Digestive Surgery, Hospital Quironsalud Sagrado Corazón (Sevilla), Seville, Spain

10 Department of Surgery, Maria Middelares Hospital, Ghent, Belgium

11 Northern Devon Healthcare NHS Trust, Barnstaple, UK

12 Department of Surgery, Wilhelmsburger Hospital Gross Sand, Academic Teaching Hospital of University Hamburg, Hamburg, Germany

13 Department of Surgery, OLVG Hospital, Oosterpark 9, 1091 AC Amsterdam, The Netherlands

14 Department of General Surgery, Royal Infirmary of Edinburgh, Edinburgh, UK 


\section{General surgical principles in the emergency setting}

During this pandemic, when dealing with an acutely complicated hernia (incarcerated/strangulated), those patients identified as COVID-19 positive are very likely to be true positive. However, the COVID-19 status of those not tested (but assessed on their current symptoms) or tested negative does not mean that they are truly COVID-19 negative. Therefore, great care should be taken to minimize the risk of infection to healthcare workers. This includes personal protection equipment and controlling any aerosol of bodily fluids which might contain the virus during surgery.

As a general concept, surgical technique and materials do not change as a consequence of the pandemic. However, some of the principles of abdominal wall surgery need clarification as a result of recent criticism (mesh, minimally invasive surgery) and adaptation of technique (midline restoration) to minimise raising intra-abdominal pressure.

The aims of emergency hernia surgery can be broken down into three parts: save life (resect dead bowel/tissue); restore GI continuity (if bowel resection undertaken); and repair the abdominal wall. These aims may be achieved at the same operation, but may require a staged approach. Mortality and morbidity increase in the emergency setting by $10-20$-fold compared to elective surgery, with bowel infarction the major risk factor for this [1].

In the absence of suspected strangulated tissue in the hernia, the use of taxis (manual reduction of the incarcerated hernia) under sedation/analgesia followed by observation may allow surgery to be safely delayed for a number of weeks [2]. This is less likely to be successful for femoral hernias.

\section{The use of mesh in the emergency setting?}

Currently, there is no evidence contraindicating the use of a mesh in a COVID-19 patients. The EHS favors mesh implantation in hernia surgery for the associated reduced risk of recurrence. In particular:

\section{Groin hernias}

- Clean and clean-contaminated cases: the use of mesh is favored similar to elective hernia repair

- Contaminated cases: monofilament large pore polypropylene mesh is suggested

- Dirty cases: it is suggested not to use mesh

\section{Quality of the available evidence: LOW}

\section{Analyzed in guidelines: Herniasurge [3]}

\section{Primary ventral and incisional hernias}

It is suggested that the emergency repair of umbilical or epigastric hernias should be tailored to the patient and hernia characteristics. The use of mesh should be considered in clean and clean-contaminated cases.

Quality of the available evidence: $L O W$

Analyzed in guidelines: EHS and AHS guidelines on umbilical and epigastric hernias [4]

\section{Is anatomical reconstruction of the abdominal wall (midline primary and incisional hernias) a priority in emergency repair?}

Current expert opinion favors extraperitoneal mesh placement and midline reconstruction over bridging with intraperitoneal mesh. These two principles should be weighed against the clinical condition of the patient, the presence of COVID-19 infection and the current or possible need for further mechanical lung ventilation. The longer the operation, the increased anesthetic pulmonary barotrauma, and the possible increased risk of a worse outcome if the patient manifests COVID-19 infection in the post-operative period.

In case of a large defect $(>10 \mathrm{~cm})$, every effort should be made to minimize any significant increase in intra-abdominal pressure at the end of the procedure-using a bridged repair may be the correct strategy in these cases.

Considering the higher risk of Surgical Site Occurrence (SSO) and SSOPI (Surgical Site Occurrence requiring Procedural Intervention), component separations (anterior and posterior) should be used wisely, balancing the risk of adverse events with that of recurrence.

Available References [5, 6]

\section{Is minimal invasive surgery safe in the emergency setting?}

While laparoscopic surgery was highly feared at the beginning of the pandemic $[7,8]$, the evidence to date does not suggest an increased risk of COVID-19 transmission during laparoscopic surgery when compared to open surgery.

SARS-CoV-2 is transmitted by aerosol droplets released by breathing, coughing, and sneezing. It has been identified in other bodily fluids: in feces $(23-82 \%$ of cases persisting for days after sputum negative [9]), blood 1-15\% [10], and no evidence concerning the peritoneal cavity per se. On the other hand, studies have shown that similar virus can be retrieved in surgical smoke (HBV, HIV, and HPV) [11], so great care should be taken to reduce aerosolisation and direct contamination. The risk of contagion is unlikely when 
general prevention strategies have been applied as recommended by the other societies (SAGES-EAES [12], ACS):

Based on these, we can conclude with some general recommendations:

1. There is no evidence for contraindication of the laparoscopic approach. In fact, the laparoscopic approach seems to allow better control of surgical smoke/plume than laparotomy [12].

2. There is little-to-no evidence of infection via blood transmission or as a consequence of a pneumoperitoneum per se from patient to healthcare worker [13]. Nevertheless, some additional factors seem sensible to introduce with the current level of knowledge.

3. COVID-19 is found in the stool of a proportion of patients [9]. Therefore, in any surgery in which the bowel is opened, regardless whether laparo/endoscopic or open, care should be taken because of the presumed risk of virus exposure from the intestinal content.

4. The desufflation of any gas from the pneumoperitoneum through a virus filter is recommended. Consider also to fully desufflate the abdomen BEFORE extracting any tissue specimen [12], Also take care when inserting equipment such as mesh or sutures through a port, as this will temporarily disrupt the valve allowing gas escape.

5. Wear Personal Protective Equipment (PPE) as for local current guidelines in all procedures involving COVID19 positive or suspected patients.

6. Surgeons should choose energy devices with care, as well as in open surgery, avoiding those which produce more particles (for example, ultrasonic devices) [14].

7. Trocar wounds should be kept at minimum length to avoid air leaks around the port. In the case of openaccess trocars, the use of ports with retention balloon should be considered to minimize inadvertent removal of the port [12].

The use of laparoscopy should be balanced taking into account the risks and benefits. Laparoscopy has clear benefits to the patient in terms of wound management, mobilization, and discharge over open surgery. As in non-COVID-19 times, the need for general anesthesia, the operative time, the expected morbidity, and surgeons' experience should be considered for every patient.

Chinese guidelines published in February, on the other hand, warn against other issue connected with laparoscopic procedures-increased intra-abdominal pressure and a potential "double" lung injury from the viral injury and a need of higher ventilation pressures due to increased intraabdominal pressure during laparoscopy. No other guideline discusses this aspect, and whether this is a theoretical or practical risk is unclear at present.
Some laparoscopic procedures on the abdominal wall are time-consuming and have longer learning curve. To utilize the benefits of laparoscopy and minimize the risks, we suggest to discontinue surgical training for junior surgeons, and all surgeons learning new techniques, in emergency hernia repair in potentially COVID+ patients.

\section{Groin hernia}

Little evidence on this approach compared to an open approach in the emergency setting exists. The laparoscopic approach can be performed in carefully selected patients.

Quality of the available evidence: VERY LOW

Analyzed in guidelines: Herniasurge [3]

\section{Primary and incisional hernia repair}

Again, the laparoscopic approach to emergency abdominal wall hernias is feasible, but evidence is lacking of its risks and benefits compared to open surgery. The laparoscopic approach can be performed in carefully selected patients.

Quality of the available evidence: LOW

Analyzed in guidelines: EHS and AHS guidelines on umbilical and epigastric hernias [4]

Other references [15]

\section{General principles in the elective setting}

\section{Can hernia repair be safely postponed until after the pandemic has passed?}

Watchful waiting is a term used for a non-operative approach and long-term monitoring of patients with various hernias and also currently our only source of reliable information on the safety of delayed surgery. During the COVID pandemic, we are not talking about WW over years, but rather a delayed or postponed operation, by several months and in some countries perhaps just weeks. The risks mentioned in papers dedicated to WW are, therefore, exaggerated when applied to the current situation and should be taken more as a surrogate indicator of relative risks.

Published guidelines support watchful waiting (WW) as safe especially in asymptomatic or minimally symptomatic patients.

\section{Groin hernias}

The probability of requiring emergency repair for male patients with primary inguinal hernia submitted to $\mathrm{WW}$ is very low (around $0.2 \%$ per year in a long-term RCT [16]). Currently, no evidence exists in favor of WW for symptomatic male patients. WW is less easy to advise on in the 
case of females (since they present in $17 \%$ of cases with acute complications) and femoral hernias (36-39\% acute presentation). Nevertheless, these figures for females include emergency presentation with a new diagnosis, and the acute risk in females with a known diagnosis of a groin hernia will be less than this over a several month postponement period.

Risk factors for emergency surgery: patients with a history of frequent access to emergency services for pain, longer symptom duration, advanced age, femoral hernia, and female sex.

Quality of the available evidence: $H I G H$

Analyzed in guidelines: Herniasurge [3]

Other references [17]

\section{Primary ventral and incisional hernias}

The probability of requiring emergency repair in WW patients is again low $(0.8 \%$ for incisional and $0.8 \%$ for umbilical/epigastric hernias at 1 year).

Quality of the available evidence: LOW (1 trial)

Analyzed in guidelines: EHS and AHS guidelines on umbilical and epigastric hernias [4]

Other references [18]

Special conditions (lateral abdominal wall defects, parastomal hernias, and hernias in defined clinical scenarios)

There is no direct evidence on the comparative outcome of the benefit of watchful waiting versus surgery for these categories. The EHS recommends the adoption of a cautious policy: consider the balance between risks and benefits, ideally postponing the procedure as far as possible until the end of the pandemic.

Quality of the available evidence: very low

Analyzed in guidelines: HerniaSurge [3]; EHS and AHS guidelines on other primary ventral hernias [19]; EHS guidelines on Parastomal Hernia [20]

\section{Trusses and binders for symptom relief and prevention of acute events}

Inguinal binders are discouraged by some guidelines, and of note, they were not considered as part of the treatment strategy during watchful waiting strategy in the HerniaSurge guidelines. The evidence on their effectiveness is old, conflicting, and sparse. Their use is not encouraged, but some patients do receive benefit in the short term and their use is not unreasonable.

A similar concept applies to incisional and ventral hernias: the pre-operative use of binders is poorly explored, and no recommendation can be made on these devices. Again, a trial of a binder is not unreasonable and continuing its use if it provides symptom relief.

There is no evidence that they can prevent acute hernia events.
Quality of the available evidence: VERY LOW

Useful references: [21-23]

\section{What information should be given to patients on the waiting list during the COVID-19 pandemic?}

It is important that all patients waiting for elective surgery should be reassured that in most cases, the delay in hernia repair will not cause any increased risk of complication.

They should also be informed of the reasons to delay their hernia repair:

1. Patients (and/or their relatives) may have undiagnosed COVID-19 infection and may develop severe disease during hospital stay and/or transmit infection to care staff.

2. The need to take care of patients suffering COVID-19 infection, both critically and non-critically ill.

3. The limited resources in terms of healthcare workers and medical equipment that may well be required to treat patients with COVID-19 infection.

4. Advice on symptoms and signs of concern relating to impending strangulation or bowel obstruction should be given, although no standardized questionnaire has been developed and validated to our knowledge. Constant pain at the site of the hernia, red overlying skin, tender to touch, nausea, and vomiting along with abdominal distension are symptoms of concern.

A program of online or telephone consultation should be provided if possible to help manage this group of patients. It is good practice to make an entry in the medical record of the patient contact. It is also useful to provide a contact number, even if only operational during office hours for patients on the waiting list to be able to seek medical advice if there are significant changes in their hernia symptoms.

\section{Follow-up of patients following hernia surgery during the COVID-19 pandemic?}

Access to follow-up clinic in terms of transport and clinic availability is an issue. Currently, there are a number of reports relating to post-operative interventions in abdominal wall surgery showing promising results on recurrence detection [24], wound complications [25], and patientreported outcomes [26] using smartphone technology. These could be useful to reduce the need for supplementary access to the hospital after hernia repair and help screen those who do require to be seen. 


\section{Which patients should be operated upon first after lockdown measures eased?}

Elective surgery will be rescheduled depending on the end of quarantine measures and both patient and hernia characteristics. Information on self-isolation prior to surgery along with the potential for pre-operative COVID19 testing depending on the local situation at the time is suggested.

A risk stratification for hernia complication (incarceration/strangulation) is suggested to be undertaken in order to minimize the need for emergency repair. But a painful hernia, or one that is becoming more difficult to reduce, should receive priority.

There will be pressure on hospitals at this time, and thus seeking to avoid surgery on those most at risk of complications after surgery seems sensible. Several tools to predict individual risk of complications are available.

- ACS-NSQIP Risk Calculator [27] (and the derived versions), even if not hernia specific, has been validated in abdominal wall surgery and is able to predict 30-day-morbidity, mortality, length of stay, and ICU requirement.

- VHRS [28] and HWRAT [29] scores are able to predict the risk of surgical site occurrences and were validated in external trials.

These data can be integrated with the risk of acquiring in-hospital COVID-19 infection and the related outcomes (mortality, ICU admission) which will vary depending on the prevalence of COVID-19 infection at the time. Complex abdominal wall surgery should be evaluated in a multidisciplinary team setting where possible.

\section{Groin hernias' prioritization}

1. Complicated: mesh-related infections, and moderate and above symptomatic recurrences in patients with low risk of developing severe complications in case of COVID19 infection

2. Risk of acute complications: patients highly symptomatic and/or with groin pain or impairment of daily activity (in particular those patients experiencing frequent attendances/admissions for pain), symptom duration, age, and hernia site (femoral)

3. Mild symptomatic/asymptomatic, frail patients.

Quality of the available evidence: LOW

Analyzed in guidelines: HerniaSurge [3]
Primary ventral and incisional hernias prioritisation

1. Complicated: mesh-related infections, moderate and above symptomatic recurrences, and staged procedures in patients with low risk of developing severe complications in case of COVID-19 infection.

2. Risk of acute complications: patients highly symptomatic + older patients having high BMI, chronic diseases, and hernia width greater than $3-4 \mathrm{~cm}$ in diameter (in periumbilical region); multi-recurrent.

3. mild symptomatic /asymptomatic; frail patients.

Quality of available evidences: LOW

Useful references [30-32]

\section{General surgical principles in the elective setting (after lockdown measures eased)}

The number one issue after lockdown measures ease will be the selection of patients that need to prioritized for early surgery and who can wait longer. However, elective abdominal hernia repair should follow current recommendations and guidelines.

Based on your local situation, evaluate the need for general anesthesia and adoption of local and regional techniques to reduce ventilator requirement, inpatient stay, and potential lung injury.

Try to avoid post-operative ICU admission and reduce the risk of adverse events and post-operative hospital stay:

- Pre-habilitation pathway (smoke cessation 4-6 weeks prior to surgery, weight loss above $35 \mathrm{~kg} / \mathrm{m}^{2}$ ) are encouraged Quality of available evidences: LOW

- Consider the addition of botulinum toxin type A for hernias with loss of domain; however, we acknowledge that botox is not registered for this purpose in many countries

Quality of available evidences: LOW.

- Consider laparoscopy to reduce the occurrence of wound events; laparoscopy has proven to be superior to open surgery in terms of post-operative infections in the treatment of inguinal and primary ventral hernias when undertaken by surgeons trained in these techniques, but this should be balanced against the need for general anesthesia.

Quality of available evidences: LOW (in subgroup analysis)

Quality of available evidences: LOW

Analyzed in guidelines: HerniaSurge [3]; EHS and AHS guidelines on umbilical and epigastric hernias [4];

IEHS guidelines update [33] 


\section{Conclusion}

Recommendations on the treatment of hernia patients during the COVID pandemics will vary among countries with different level of outbreak, resource utilization for COVID patients, local testing capacities, and availability of PPE. Regardless of these geographical differences, it is important to remember that hernia surgery is, in most cases, a procedure that can be postponed and is in an elective setting a quality of life increasing not life saving procedure.

Today, more than yesterday, it is time to revise some general principles for emergency hernia surgery. During any operation, extra attention has to be paid to minimizing the risk of virus spread and lung injury, and shortening the hospital stay through adapting and tailoring our surgical approach to the patient, the situation, and the surgeon variables.

Funding No funding requested or given.

\section{Compliance with ethical standards}

Conflict of interest None of the authors has competing interests with the present document.

Ethics approval Since the nature of the guidance, no ethical approval was requested.

Informed consent For the present type of study formal consent is not needed.

\section{References}

1. Duan SJ, Qiu SB, Ding NY, Liu HS, Zhang NS, Wei YT (2018) Prosthetic mesh repair in the emergency management of acutely strangulated groin hernias with grade I bowel necrosis: a rational choice. Am Surg 84(2):215-219

2. Harissis HV, Douitsis E, Fatouros M (2009) Incarcerated hernia: to reduce or not to reduce? Hernia 13(3):263-266. https://doi. org/10.1007/s10029-008-0467-9

3. HerniaSurge $\mathrm{G}$ (2018) International guidelines for groin hernia management. Hernia 22(1):1-165. https://doi.org/10.1007/s1002 9-017-1668-x

4. Henriksen NA, Montgomery A, Kaufmann R, Berrevoet F, East B, Fischer J, Hope W, Klassen D, Lorenz R, Renard Y, Garcia Urena MA, Simons MP, European and Americas Hernia Society (2020) Guidelines for treatment of umbilical and epigastric hernias from the European Hernia Society and Americas Hernia Society. Br J Surg 107(3):171-190. https://doi.org/10.1002/bjs.11489

5. Deerenberg EB, Timmermans L, Hogerzeil DP, Slieker JC, Eilers PH, Jeekel J, Lange JF (2015) A systematic review of the surgical treatment of large incisional hernia. Hernia 19(1):89-101. https:// doi.org/10.1007/s10029-014-1321-x

6. Alkhatib H, Tastaldi L, Krpata DM, Petro CC, Olson M, Rosenblatt S, Rosen MJ, Prabhu AS (2019) Outcomes of transversus abdominis release in non-elective incisional hernia repair: a retrospective review of the Americas Hernia Society
Quality Collaborative (AHSQC). Hernia 23(1):43-49. https:// doi.org/10.1007/s10029-019-01878-z

7. Di Saverio S, Khan M, Pata F, Ietto G, De Simone B, Zani E, Carcano G (2020) Laparoscopy at all costs? Not now during COVID19 and not for acute care surgery and emergency colorectal surgery: a practical algorithm from a Hub Tertiary teaching hospital in Northern Lombardy. Italy. J Trauma Acute Care Surg. https:// doi.org/10.1097/ta.0000000000002727

8. Zheng MH, Boni L, Fingerhut A (2020) Minimally invasive surgery and the novel coronavirus outbreak: lessons learned in China and Italy. Ann Surg. https://doi.org/10.1097/sla.000000000000392 4

9. Tian Y, Rong L, Nian W, He Y (2020) Review article: gastrointestinal features in COVID-19 and the possibility of faecal transmission. Aliment Pharmacol Ther 51(9):843-851. https://doi. org/10.1111/apt.15731

10. Wang W, Xu Y, Gao R, Lu R, Han K, Wu G, Tan W (2020) Detection of SARS-CoV-2 in different types of clinical specimens. JAMA. https://doi.org/10.1001/jama.2020.3786

11. Alp E, Bijl D, Bleichrodt RP, Hansson B, Voss A (2006) Surgical smoke and infection control. J Hosp Infect 62(1):1-5. https://doi. org/10.1016/j.jhin.2005.01.014

12. Francis N, Dort J, Cho E, Feldman L, Keller D, Lim R, Mikami D, Phillips E, Spaniolas K, Tsuda S, Wasco K, Arulampalam T, Sheraz M, Morales S, Pietrabissa A, Asbun H, Pryor A (2020) SAGES and EAES recommendations for minimally invasive surgery during COVID-19 pandemic. Surg Endosc. https://doi. org/10.1007/s00464-020-07565-w

13. Bree K, Barnhill S, Rundell W (2017) The dangers of electrosurgical smoke to operating room personnel: a review. Workplace Health Saf 65(11):517-526. https://doi.org/10.1177/2165079917 691063

14. Choi SH, Kwon TG, Chung SK, Kim TH (2014) Surgical smoke may be a biohazard to surgeons performing laparoscopic surgery. Surg Endosc 28(8):2374-2380. https://doi.org/10.1007/s0046 4-014-3472-3

15. Awaiz A, Rahman F, Hossain MB, Yunus RM, Khan S, Memon B, Memon MA (2015) Meta-analysis and systematic review of laparoscopic versus open mesh repair for elective incisional hernia. Hernia 19(3):449-463. https://doi.org/10.1007/s 1002 9-015-1351-z

16. Fitzgibbons RJ Jr, Ramanan B, Arya S, Turner SA, Li X, Gibbs JO, Reda DJ, Investigators of the Original Trial (2013) Long-term results of a randomized controlled trial of a nonoperative strategy (watchful waiting) for men with minimally symptomatic inguinal hernias. Ann Surg 258(3):508-515. https://doi.org/10.1097/ SLA.0b013e3182a19725

17. Dahlstrand U, Wollert S, Nordin P, Sandblom G, Gunnarsson U (2009) Emergency femoral hernia repair: a study based on a national register. Ann Surg 249(4):672-676. https://doi. org/10.1097/SLA.0b013e31819ed943

18. Kokotovic D, Sjolander H, Gogenur I, Helgstrand F (2016) Watchful waiting as a treatment strategy for patients with a ventral hernia appears to be safe. Hernia 20(2):281-287. https://doi. org/10.1007/s10029-016-1464-Z

19. Henriksen NA, Kaufmann R, Simons MP, Berrevoet F, East B, Fischer J, Hope W, Klassen D, Lorenz R, Renard Y, Garcia Urena MA, Montgomery A, on behalf of the European Hernia Society, the Americas Hernia Society (2020) EHS and AHS guidelines for treatment of primary ventral hernias in rare locations or special circumstances. BJS Open 4(2):342-353. https://doi.org/10.1002/ bjs5.50252

20. Antoniou SA, Agresta F, Garcia Alamino JM, Berger D, Berrevoet F, Brandsma HT, Bury K, Conze J, Cuccurullo D, Dietz UA, Fortelny RH, Frei-Lanter C, Hansson B, Helgstrand F, Hotouras A, Janes A, Kroese LF, Lambrecht JR, Kyle-Leinhase 
I, Lopez-Cano M, Maggiori L, Mandala V, Miserez M, Montgomery A, Morales-Conde S, Prudhomme M, Rautio T, Smart N, Smietanski M, Szczepkowski M, Stabilini C, Muysoms FE (2018) European Hernia Society guidelines on prevention and treatment of parastomal hernias. Hernia 22(1):183-198. https:// doi.org/10.1007/s10029-017-1697-5

21. Cheek CM, Williams MH, Farndon JR (1995) Trusses in the management of hernia today. Br J Surg 82(12):1611-1613. https://doi. org/10.1002/bjs. 1800821207

22. Whitaker J, Akritidis G, Baker D (2014) Who's trusting in a truss? Hernia 18(1):147-148. https://doi.org/10.1007/s 1002 9-013-1191-7

23. Strigård K, Stark B, Bogren A, Gunnarsson U (2015) Ventral hernia and patient experience of an elastic girdle. ANZ J Surg 85(7-8):525-528. https://doi.org/10.1111/ans.12924

24. van den Heuvel B, van Jarwaarde JA, Wichers P, de Lange de Klerk ES, Bonjer HJ, Dwars BJ (2015) Follow-up after laparoscopic inguinal hernia repair, can it be done by phone? A prospective study in 300 patients, the PINQ-PHONE. Surg Endosc 29(11):3292-3297. https://doi.org/10.1007/s00464-015-4080-6

25. Kummerow Broman K, Gaskill CE, Faqih A, Feng M, Phillips SE, Lober WB, Pierce RA, Holzman MD, Evans HL, Poulose BK (2019) Evaluation of wound photography for remote postoperative assessment of surgical site infections. JAMA Surg 154(2):117124. https://doi.org/10.1001/jamasurg.2018.3861

26. van der Meij E, Anema JR, Otten RHJ, Huirne JAF, Schaafsma FG (2016) The effect of perioperative e-health interventions on the postoperative course: a systematic review of randomised and non-randomised controlled trials. PLoS One 11(7):e0158612e0158612. https://doi.org/10.1371/journal.pone.0158612

27. ACS-NSQIP Surgical Risk Calculator. https://riskcalculator.facs. org/RiskCalculator/index.jsp. Accessed 01 Apr 2020

28. Berger RL, Li LT, Hicks SC, Davila JA, Kao LS, Liang MK (2013) Development and validation of a risk-stratification score for surgical site occurrence and surgical site infection after open ventral hernia repair. J Am Coll Surg 217(6):974-982. https://doi. org/10.1016/j.jamcollsurg.2013.08.003
29. Fischer JP, Wink JD, Tuggle CT, Nelson JA, Kovach SJ (2015) Wound risk assessment in ventral hernia repair: generation and internal validation of a risk stratification system using the ACSNSQIP. Hernia 19(1):103-111. https://doi.org/10.1007/s1002 9-014-1318-5

30. Sneiders D, Yurtkap Y, Kroese LF, Kleinrensink GJ, Lange JF, Gillion JF (2019) Risk factors for incarceration in patients with primary abdominal wall and incisional hernias: a prospective study in 4472 patients. World J Surg 43(8):1906-1913. https:// doi.org/10.1007/s00268-019-04989-x

31. Shubinets V, Carney MJ, Colen DL, Mirzabeigi MN, Weissler JM, Lanni MA, Braslow BM, Fischer JP, Kovach SJ (2018) Management of infected mesh after abdominal hernia repair: systematic review and single-institution experience. Ann Plast Surg 80(2):145-153. https://doi.org/10.1097/sap.0000000000001189

32. Lau B, Kim H, Haigh PI, Tejirian T (2012) Obesity increases the odds of acquiring and incarcerating noninguinal abdominal wall hernias. Am Surg 78(10):1118-1121

33. Bittner R, Bain K, Bansal VK, Berrevoet F, Bingener-Casey J, Chen D, Chen J, Chowbey P, Dietz UA, de Beaux A, Ferzli G, Fortelny R, Hoffmann H, Iskander M, Ji Z, Jorgensen LN, Khullar R, Kirchhoff P, Kockerling F, Kukleta J, LeBlanc K, Li J, Lomanto D, Mayer F, Meytes V, Misra M, Morales-Conde S, Niebuhr H, Radvinsky D, Ramshaw B, Ranev D, Reinpold W, Sharma A, Schrittwieser R, Stechemesser B, Sutedja B, Tang J, Warren J, Weyhe D, Wiegering A, Woeste G, Yao Q (2019) Update of Guidelines for laparoscopic treatment of ventral and incisional abdominal wall hernias (International Endohernia Society (IEHS)): part B. Surg Endosc 33(11):3511-3549. https://doi. org/10.1007/s00464-019-06908-6

Publisher's Note Springer Nature remains neutral with regard to jurisdictional claims in published maps and institutional affiliations. 